\title{
EFFECT OF THERAPEUTIC EXERCISES ON SOME SELECTED DRUGS
}

\author{
Journal website at; \\ http://mrtbjournal.org/index.php/njmr/issue/current/showToc
}

\author{
ONIGBINDEAT,OYERINDE O*,TALABIAE*,OBIYEMIOO*,OGUNSAKINEA*, \\ ADESOYEA.A*, SHEU RA*, ONIYANGI SO*, ORLJAJOGUNOO** \\ Medical Rehabilitation Department, Obafemi Awolowo University, Ile-Ife, Osun State, Nigeria \\ *Human Kinetics and Health Education Dept, University of Ilorin, Ilorin, Kwara State. \\ **Physiotherapy Depatment, State Hospital Iwo
}

\author{
Correspondence to: \\ ayotesonigbinde@yahoo.co.uk
}

\section{SUMMARY}

Therapeutic exercise is not just an exercise, it is a structured and dosage specific physical activity. Exercise is being used simultaneously with drugs and it has been observed to have influence on the pharmacokinetic of drugs. Similarly, most individual utilizes both exercise and drugs to maintain good health and physical fitness. Exercises and drugs play important roles in control of pain, obesity, weakness, protection against degenerative diseases, improving cardiovascular endurance and respiratory efficiency. Just like drugs, it stimulates a healing response that couples both inflammatory and antiinflammatory mechanisms to repair, regenerate, and grow stronger tissues. There are evidences that both drugs and exercise play the same roles in some diseases; hence, there is likelihood of interactions in the physiological responses of the body. Exercise is accessible, safe, and an inexpensive anti-inflammatory medicine and it also has web-like interactions in the body unlike drugs that has a single targeted action. Physiotherapists utilize exercises in the management of conditions such as osteoarthritis, stroke, hypertension, diabetes and cardiac diseases; and most of the subjects are equally on drugs. This review showed that exercises affect pharmacokinetic variables of drugs resulting in either positive or negative influence depending on the type of drugs, exercises intensity, duration and excretory organ.

Keywords: Therapeutic exercise, Drugs, pharmacokinetic variables, exercises intensity

\section{INTRODUCTION}

Therapeutic exercise is an invention encompassing a broad range of activities designed to restore or improve musculoskeletal, cardiopulmonary and or neurological function (ENAH 2007). Exercise is physical activity that is planned, structured, and repetitive for the purpose of conditioning any part of the bodys and improve fitness (Health A to Z 1998). Good health provides foundation for which fitness rests and at the same time fitness provides one of the most important keys to grow healthy (Jatau 2000). It is useful in preventing or treating coronary heart disease, Osteoporosis, weakness, diabetes, Obesity and depression. American Health association reported that exercise was beneficial for patients' awaiting heart transplant, and that it also reduces risk of women in developing breast cancer (Health A to Z, 1998).

Exercise is a readily accessible, safe, and an inexpensive anti-inflammatory medicine (Jade, 2006). It also requires supervision with dose specificity. When exercise is in adequate form, it stimulates a healing response that couples both inflammatory and anti-inflammatory mechanisms to repair, regenerate, and grow stronger issue (Jade, 2006). Drugs have single targeted action while exercise has web-like interactions in the body (Jade, 2006).

The exercise capacity of patients with atherosclerotic coronary heart disease appears to be significantly altered by certain cardiovascular drugs, alone or in combination. This can be attributed to complex interactions of homodynamic and electrophysiological change (Wenger, 1985). The greatest benefit occurs as muscles improve the efficiency of their oxygen use, which reduces the need for the heart to pump more blood. The relative increase in endurance following exercise should also translate into a generally more active lifestyle (Health A to $Z, 1998$ ). Physical activity is a key component of energy balance and is promoted in children and adolescents as a lifelong positive health behavior. The potential behavioral 
determinants necessitate understanding influences from three fundamental areas: 1) physiologic and developmental factors, 2) environmental factors, and 3) psychological, social, and demographic factors (Harold et al, 1998).

Most times, exercise is being used simultaneously with drugs and it has been observed to have influence on the pharmacokinetic of drugs (Onigbinde et al. 2006). Pharmacokinetic is an area of pharmacology that deals with drug administration, absorption, distribution, tissue building, metabolism and excretion (Ciccone 1995). Variables associated with pharmacokinetic determines the specificity of adequate dose of the drugs that will reach the target organ and exert a response (Onigbinde et al, 2006). Various scientists had documented the efficiency of electromotive and mechanical force (exercise inclusive) in delivering pharmacological agents (kari, 1986, Kim et al, 1993, KAHFS, 1996)/Baixauli et al (1990) and Khazaeinia et al (2000) observed that exercise and massage influenced normal pharmacokinetic. Other established factors that affect drug response are body weight, age, sex, route of administration, emotional factors time of administration, pre- existing disease and patients' drug history (Gerald 1981).

Exercise increases bioavailability because it increases tissue heat during exercises, hence, resulting in increase in kinetic molecular movement. There is confusion in various reports on the effect of exercises on bioavailability (Khazemia et al, 2000, sarwrymiowez, chockspolly 1997). However, effect of exercises on orally administered drug depends on intensity; duration and type of exercise bout (Dyke et al, 1998).

Absorption is generally increased in subcutaneous or intramuscular injection; when the injected site is exercised. The increased bioavailability has clinical implication because drugs are readily made available at the target tissues. The significant effect of exercise has been well noted on insulin. When precaution is not taken, the subject goes into hypoglycemic shock because the exercise will regularly make available the insulin drugs which resultantly increase the consumption of glucose in the body. Dyke et al (1998) reported that excessive decreases splanic blood flow, limit blood to the liver, hence, decreases the hepatic clearance of certain drugs.

Physiotherapists, exercise physiologists and health educationists utilize exercise prescription for conditions such as osteoarthritis, stroke, hypertension, diabetes and cardiac diseases; and most of the subjects are equally on drugs but it appears little is still understood about the joint effect of exercises and drugs. The explanation and knowledge on relative interaction of drugs with exercises is still scanty hence the need for this review.

\section{EFFECT OFEXERCISEANDENVIRONMENTALHEAT ONDRUG}

The effect of physical exercise and heat exposure on the pharmacokinetic behaviour of some model drugs was investigated in normal volunteers by (Danon. 1984). In the study, exercise was mild to moderate and environmental temperature was either $22 \mathrm{C}$ or $40 \mathrm{C}$. Under heat exposure and exercise, theophlline exhibited prolonged half-life and reduced clearance. This is suggesting the need for dosage adjustment in moderately active individuals. Exercise influenced digoxin kinetics by shortening the time to peak, possibly enhancing the drug's absorption. The plasma kinetics of quanidine did not change under exercise conditions. However, urinary excretion of the drug was significantly higher during exercise than at rest, and this correlated with increased urinary output during exercise. Exercise and heat exposure prolonged the time of distribution of intravenously administered propanolol, while other pharmacokinetic parameters relating to propanolol remained. The half lives (T1/2) of both sulfamethoxazole and trimethoprin that were administered as co-trimoxazole were not significantly altered.

\section{EFFECTSOFEXECISES ONSELECTEDDRUGS}

Khazaenia (2002) reported that exercise does not have substantial effect on the absorption of orally administered drugs but aids absorption from intramuscular, subcutaneous, transdermal and inhalation site. The relative effect of exercises depends on the types of drug:

\section{Digoxin}

Digoxin is a drug of choice for heart failure. Matsuda et al (1991) in an experimental study found that digoxin was effective in reducing heart rate at rest but failed to reduce it during exercise. Exercise increases muscular blood flow, hence, there is increase in the binding of digoxin with working skeletal muscles and the resultant effect is an increase in volume of distribution. In patients with moderate to severe airway obstruction due to congestive heart failure, Coates et al (1982) concluded that digoxin did not increase exercise capacity or improve exercising cardiac function.

Hall et al (1989) evaluated the effect of 1 hour of everyday exercise (walking at patient's own pace) on serum digoxin concentrations. There were three continuous phases: 1 hour of rest, 1 hour of exercise, and a final hour of rest. Serum digoxin concentrations were drawn every 20 minutes. During the first rest period, serum digoxin concentrations rose $30 \%$ from the first concentration drawn in the study. After 1 hour of exercise, serum digoxin concentrations fell $26.8 \%$ from the last concentration of the first rest period. At the end of the second hour of rest, serum digoxin concentrations increased by $36.6 \%$ from the last concentration. This showed significant changes $(\mathrm{P}<0.01)$ in concentrations at each phase of the study; hence, they concluded that the influence of everyday exercise should be taken into account when interpreting serum digoxin concentrations.

\section{Propanolol}

Bet (B) blocker drugs are used in controlling hypertension and angina; and are also helpful in preventing 
further cardiac incidents for people who have already suffered one heart attack (stansfeld, 2007). Exercise reduces the availability of propanolol in muscle, hence, reducing the availability for elimination. Generally, exercise reduces renal blood flow, hence increasing the plasma concentration of this class of drugs because they will not be available for elimination by the kidney (khazaeinia et al, 2002). Matsuda et al (1991) reported that propanolol and verapamil reduced heart rate at all levels of exercise as well as at rest. Similarly, they reported that during exercise oxygen uptake (total exercise capacity) was not reduced with propanolol or verapamil and this was attributed to have been accomplished by an increased oxygen pulse.

\section{Acetyl salicylic Acid (ASA)}

Sawrymiowez (1997) observed that there is no need for dose adjustment for subjects on ASA who moderately exercised. Hepatic blood flow decreases progressively as exercise intensity increases. A decrease in hepatic perfusion will not be affected greatly during exercise because clearance depends on the metabolic capacity of the liver rather than on the hepatic blood flow.

\section{Non steroidal anti-inflammatory drugs (NSAIDs)}

These are varieties of drugs used as analgesics and to subdue inflammatory process. The use of NSAIDs before and during exercise has been causally linked to acute renal failure in case study reports (Kenney, 2007). Excessive NSAID use increases the potential for renal problems associated with exercise (Kenney, 2007). This potential is magnified if prolonged exercise is combined with severe heat stress and dehydration. The effect on the kidney can be reduced if proper hydration is encouraged. The main side effects of NSAIDs include decreased hemostasis by restricting platelet aggregation, interfering with antihypertensive medication such as Diuretics or B-adrenceptor blockades. In some patients who have liver disease, dehydration, heart failure, or cirrhosis of the liver can potentially cause acute renal failure. NSAIDs also result in erosion and ulceration of the upper gastrointestinal tract, and can result in hemorrhage and perforation. Hung, (2002) reported that in order to maintain the plasma concentration of a drug, consideration should be given to alternative drugs if the patient is on intermittent or irregular exercise.

The absorption of methylsalicylate, when applied on the skin, was markedly influenced thus, the amount of salicylate that was absorbed increased three fold while the subjects were exposed to either heat or exercise or both (Danon, 1984)

\section{Calcium drugs and Vitamin}

The skeleton contains $99.5 \%$ of total body calcium. Sufficient calcium is vital to the formation of healthy bone (Medscape General Medicine. 1999). Brath et al (2003) found that women who are actively involved in active exercise and on Calcium drugs and Vitamin had a significant increase in their Bone Mass Density (BMD). Similarly, Zanke et al (2003) observed higher bone density amongst gymnast compared to the untrained ones. Some studies however showed that intensive physical activity could result in bone loss (Kasis and Igbesis, 2003).

There appears to be a synergistic effect of exercise and calcium on trabecular bone in postmenopausal women (Medscape General Medicine. 1999). This combination of exercise and calcium supplementation (1g of elemental calcium per day in the form of calcium lactate-gluconate) slowed bone loss when compared with exercise alone. It was also reported that increased exercise increases distalforearm bone density, with bone-density improvements slightly greater in the exercise group that also received calcium.

\section{Insulin}

It has been demonstrated that resistance training exercise improves insulin sensitivity in subject with Non Insulin dependent diabetes (NIDDM), (Ishis et al, 1998). Similarly, Mukine et al (1988) observed that a single bout of exercise increase the sensitivity and responsiveness of insulin stimulated glucose uptake in untrained individuals. Rossi et al (2005) reported that chronic aerobic exercise increases insulin vasodilator activity and improves endothelial function in elderly subjects.

American physiology society (2008) reported that after 6 days of treadmill exercise to burn 500 kilocalories ( $\mathrm{k} / \mathrm{cal}$ ) each day, the subjects in the negative energy balance (NEG) group, who received no energy replacement, showed a significant $(\mathrm{p}=0.037) 40 \%$ increase in insulin action (measured by glucose rate of disappearance/steady state insulin). However insulin action was unchanged in the zero energy balance group (ZERO), who were required to finish a sports drink during exercise and additional food afterward to "replace" the $500 \mathrm{k} / \mathrm{cal}$. American Physiological Society (2008) also reported that short-term exercise significantly increase insulin action, while cardiovascular disease risk factors trend downward.

Braun (2008) noted that exercise can be used to prevent or delay the transition from insulin resistance (prediabetes) to overt type- 2 diabetes, and in the optimal use of exercise to manage blood sugar in people who already have diabetes. Exercise increases insulin sensitivity of glucose uptake and glycogen synthesis equally in diabetic and control rats, but insulin responsiveness of glucose uptake was noted only in controls (Richter et al, 1985). There was increased muscle glucose uptake after exercise and that there is no need for insulin during exercise (Richter et al, 1985).

\section{CONCLUSION}

This review showed that excrcises affect pharmacokinetic variables of drugs resulting in either positive or negative influence depending on the type of drugs, exercises intensity, duration and excretory organ. Hence, physiotherapists and exercise physiologists need 
to take precaution and also widen their knowledge on pharmacology. Also, there is need for elaborate research as most studies are experimented on animals.

\section{REFERENCES}

- American Physiological Society (2008) Short-Term Exercise Significantly Increases Insulin Action, While Cardiovascular Disease Risk Factors Trend Downward. (301) 634-7209 (703) 967-2751 (cell)

- Baixauli F, Ingels F, Alcantra P et al (1990) percutaneous treatment of acute soft tissue lesions with naproxen gel and ketoprofen gel. J Int Med Res. 18: 372-378.

- Braith RW, Magyari P M et al (2003). Resistance exercise training and alendronate reverse glucocorticoids-induced osteoporosis in transplant recipients. Heart lung transplant, 22: 1082-1090.

- Braun (2008) in American Physiological Society (2008) Short-Term Exercise Significantly Increases Insulin Action, While Cardiovascular Disease Risk Factors Trend Downward. (301) 634-7209 (703) 967-2751 (cell)

- Ciccone CD (1995) Basic Pharmacokinetics and the potential effect of physical therapy interventions on Pharmacokinetic variables. Phys Ther 75: 343-351.

- Coates AL, K Desmond, MI Asher, J Hortop and PH Beaudry. The effect of digoxin on exercise capacity and exercising cardiac function in cystic fibrosis. Chest, Vol 82, 543-547, Copyright (C) 1982 by American College of Chest Physicians.

- Danon A (1984) Effect of exercise and environmental heat on drugs kinetics Accession number: ADA 145405.

- Dyke TM, Sams RA, Hinchcliff KW (1981) Intensitydependent effects of acute submaximal exercise on the pharmacokinetics of bromsulphalein in horses. Am J Vet Res. 1998 Nov; 59(11):1481-7.

- Gerald M. C (1981) pharmacology: An introduction to drugs second edition. Ung Ming-Cji, Chen Fu-An et al (2002) The effects of non-seroidal Anti-inflammatory drugs on skeletal muscles. The sports journ Vol 5 No 2.

- Hall PD, WR Garnett, KW Kolb, WL Rock, and H Stanley. The effect of everyday exercise on steady state digoxin concentrations. Copyright $(\mathcal{O} 1989$ by the American College of Clinical Pharmacology.

- Harold W. Kohl III* and Karen E. Hobbs. Development of Physical Activity Behaviors among Children and Adolescents Pediatrics Vol. 101 No. 3 Supplement March 1998, pp. 549-554.

- Health A-Z (1998) A world health at your finger tips. www.scribd.com/doc/6140410/Exercise-Therapy Prevention-and-Treatment-of-Disease. 12th january, 2008
- Jade T (2006) Exercise is medicine: the anti-inflammatory effects of high intensity exercise. Townsend letter for Doctors and patients, Nov.

- Jatau A. A (2000) Promoting health and fitness through physical exercise. Health and fitness journal international vol 1 No 1.

- KAHFS: Ketoprofen_American Hospital Formulary Servics (19896): Drug information. Bethesda, Md: American Society of Health-System Pharmacists Inc, 1416-1422.

- Kari (1986) control of blood glucose levels in alloxan-diabetic rabbits by iontophoresis of insulin. Diabetes; 35:271.221

- Kazis and Igbesia E (2003). The female athlete triad, Adolescent Medicine 14: 87-95

- Kenney W. Larry Nonsteroidal anti-inflammatory drugs and the kidney. Current sports medicine report, Volume 1, Number 3 / May, 2002.

- Khazaeinia T, Ramsey A. A, Tam Y. K (2000) The effect of exercise on Pharmacokinetic of drugs. J Pharm Pharm Sci. Sep-Dec, 3(3): 292-302.

- Kim A, Green PG, Rao G Guy RH. (1993) Convective solvent flow across the skin during iontophoresis. Pharm Res: 10:1315-1320.

- Matsuda M, Matsuda Y, Yamagishi T, Takahashi T, Haraguchi M, Tada T, Kusukawa R. Effects of digoxin, propranolol, and verapamil on exercise in patients with chronic isolated atrial fibrillation. Cardiovasc Res. 1991 Jun;25(6):453-7.

- Medscape General Medicine 1(3), 1999. (O 1999 Medscape, Exercise at Menopause: A Critical Difference Medscape General Medicine. Osteoporosis: Exercise vs Calcium Supplementation.

- Onigbinde AT, Adedoyin R A. Johnson OE (2006). Effects of physical therapy intervention on pharmacokinetic variables. A preliminary review. Nigerian Journ of Medical Rehabilitation, vol 2, No 1 (issue no 19).

- Richter EA, T Ploug and H Galbo (1985) Increased muscle glucose uptake after exercise. No need for insulin during exercise. Diabetes, Vol 34, Issue 10 1041-1048, Copyright (C) 1985 by American Diabetes Association.

- Sawrymowiez M and Chock-Spolly (1997). The effect of exercise on the Pharmacokinetic of acetaminophen and acetylsalicylic acid. Ann Acad Med Stetin; 43: 57-66

- Stansfeld Janet (2007) Beta blockers side effects and exercise. http:www.pponline.co.uk/encyc/0137.htm

- Wenger N. K (1985) Cardiovascular drugs: Effects on exercise testing and exercise training of the coronary patients. Cardiovascular Clin, 15 (2): 133-43.

- Zanker C. L, Garnon L et al (2003): Differences in bone density, body composition, physical activity and diet between child gymnast and untrained children 7-8 years of age. Journal of bone mineral research, 18, 1043-1056. 